\title{
¿QUÉ ES LA FILOSOFÍA? RESPUESTA CRÍTICA AL MATERIALISMO DE GUSTAVO BUENO
}

\author{
DANIEL MARTín SÁEZ \\ Universidad Autónoma de Madrid \\ http://dx.doi.org/10.15304/ag.39.1.5703
}

\section{Resumen}

Gustavo Bueno intentó definir la filosofía en dos obras que han marcado su sistema durante más de cuatro décadas: El papel de la filosofía en el conjunto del saber (1970) y ¿Qué es la filosofía? (1995). Planteamos una crítica global a esa definición, que consideramos incompleta, y proponemos completarla, redefiniéndola a una nueva escala. Mantenemos que su "filosofía de la filosofía" se centra en aspectos "sustanciales" ("Geometría de las Ideas"), olvidando los componentes "modales" (filosofía como actividad o modo de vida), lo que le habría llevado a una visión de la filosofía como "especialidad", en perjuicio de su cariz "imperativo", imposible de asimilar por ningún "saber" o "taller" concreto, lo que además ayudaría a explicar la racionalidad de las definiciones clásicas de filosofía.

Palabras clave: filosofía crítica, filosofía imperativa, filosofía de la filosofía, geometría de las ideas, materialismo filosófico.

\begin{abstract}
Gustavo Bueno tried to define philosophy in two works that have marked his system for more than four decades: El papel de la filosofía en el conjunto del saber (1970) y ¿Qué es la filosofía? (1995). I present a global critique of his definition, which I consider to be incomplete, and I propose to expand his definition and to redefine it under a new scale. I maintain that his "philosophy of philosophy" focuses on "substantial" facts ("Geometry of Ideas"), forgetting its "modal" components (philosophy as an activity or way of life). It would have led him to a vision of philosophy as a "specialty", at the sacrifice of its "imperative" mo-
\end{abstract}

Recibido: 27/12/2018. Aceptado: 11/06/2019. 
ment, impossible to assimilate by any concrete "knowledge" or "workshop". It also would help to explain the rationality of classical definitions of philosophy.

Keywords: critical philosophy, geometry of ideas, imperative philosophy, philosophical materialism, philosophy of philosophy.

\section{Introducción}

Gustavo Bueno intentó definir la filosofía en dos obras que han marcado su sistema, el denominado materialismo filosófico, durante más de cuatro décadas: El papel de la filosofía en el conjunto del saber (escrita en 1968 y publicada en 1970) y ¿Qué es la filosofía? El lugar de la filosofía en la educación (1995). ${ }^{1}$ No es mi intención exponer estas obras en detalle, sino plantear una crítica global, aunque meramente esbozada, a la definición que aparece en ellas, que consideramos incompleta. Para ello, proponemos una nueva definición de filosofía, aceptando su definición como momento de la nuestra, aunque redefiniéndola a una nueva escala. Mantenemos que su "filosofía de la filosofía" se centra en aspectos "sustanciales" de la actividad filosófica (entendida como "Geometría de las Ideas") y olvida sus componentes "modales" (como actividad o modo de vida, dirigida imperativamente hacia "las cuestiones más importantes"), lo que le habría llevado a una visión de la filosofía como "especialidad", en perjuicio de ese momento "imperativo", imposible de asimilar por cualquier "saber" o "taller" concreto, lo que además podría ayudar a explicar la racionalidad de las definiciones clásicas de filosofía allí criticadas.

\footnotetext{
${ }^{1}$ Bueno, Gustavo. El papel de la filosofía en el conjunto del saber. Ciencia Nueva, Madrid, 1970; Ib.: ¿Qué es la filosofía? El lugar de la filosofía en la educación. El papel de la filosofía en el conjunto del saber constituido por el saber político, el saber científico y el saber religioso de nuestra época, Pentalfa, Oviedo, 1995. Dejo a los historiadores la genealogía de estas obras, que sin duda ha de pasar por sus libros de los años cincuenta para Anaya, anteriores a su magisterio en Oviedo, donde la definición de filosofía diverge en aspectos esenciales de las ofrecidas aquí. Véase Bueno, Gustavo. "Un profesor de filosofía, autor de libros de texto, en la década del 'nacional catolicismo' español". El Catoblepas, $\mathrm{n}^{\circ} 99$ (2010), p. 2. Tampoco pretendo detenerme aquí en la importancia doctrinal de estas obras, determinada por el hecho de que sólo a partir de los años setenta cristalizó el sistema del materialismo filosófico, marcado por obras como los Ensayos materialistas (1972), El animal divino (1985) o los cinco volúmenes de la Teoría del cierre categorial (19921993). Hasta su fallecimiento en 2016, Bueno nunca dejó de remitirse a esas dos obras para defender su definición de la filosofía. E. g., Bueno, Gustavo. "Historia de las Ideas filosóficas”. El Catoblepas, n 162 (2015), p. 2.
} 


\section{Esbozo de una clasificación de acepciones de filosofía}

Para llegar a su definición, Bueno desecha varias concepciones clásicas de filosofía, que agrupa de diversas formas. Para el presente ensayo, propongo una nueva clasificación, compatible con las realizadas en dichas obras, que nos sirva para alcanzar nuestros objetivos.

Atendemos al siguiente criterio: si la filosofía se toma como una idea recta (que tendría su propio "campo", "autonomía", "contenido", etc., que sólo ella podría conocer, plantear, criticar) u oblicua (donde la filosofía estaría subordinada a otros saberes, en los que se buscaría su esencia). Las acepciones rectas son las más numerosas, aunque no necesariamente las más difundidas en la actualidad, y podemos dividirlas en acepciones "no-subjetuales" y "subjetuales", según tomen ese contenido como algo esencialmente vinculado o no a una idea de sujeto ("animal racional", "hombre", "ego", "persona”, “yo", “conciencia", "sujeto trascendental”, "alma”, etc.). Las definiciones oblicuas basta dividirlas en "ancilares" y "parasitarias", según subordinen la filosofía a otros saberes de manera parcial o total. Se podrían poner muchos más ejemplos, pero bastarán los siguientes para nuestro propósito:

ACEPCIONES ReCtAS:

Acepciones no-subjetuales:

1. Acepciones aitiológicas: filosofía como "investigación de las causas primeras" (teológicas, físicas, etc.).

2. Acepciones onto-existencialistas: filosofía como dedicación a los “eternos interrogantes de la existencia” (e.g., el Ser, la Nada, etc.).

3. Acepciones gnósticas: filosofía como saber absoluto, ya sea eterno ("plotiniano") o histórico ("hegeliano").

Acepciones subjetuales:

4. Acepciones egocéntricas: filosofía como contenido de un ego o conciencia pura (sustancial), o como estudio de ese ego.

5. Acepciones subjetivo-existencialistas: filosofía como resultado del sujeto existencial (no sustancial) que interroga.

6. Acepciones psicologistas: filosofía como amor a la sabiduría (concepción etimológica), o como producto de una especial curiosidad.

7. Acepciones antropológicas: filosofía como posibilidad del animal rationale (e. g., el niño "que alcanza la fase del por qué" como animal philosophicum, pero también el hombre primitivo - $\mathrm{O}$ 
el "presente etnológico"-, según la tesis aristotélica de la curiosidad natural como inicio del filosofar) o del animal político (e.g., la filosofía "en sentido lato" definida por Bueno, cosmovisionaria).

\section{ACEPCIONES OBLICUAS:}

8. Acepciones ancilares: subordinan la filosofía a otros saberes, prácticas, instituciones (filosofía como ancilla theologiae, scientiarum, civitatis, democratiae, universitatis, etc.), con la intención de precederlos, aclararlos, allanar su camino, etc. La "filosofía espontánea de los científicos”, o la idea de filosofía como mater scientiarum, son igualmente ancilares, al tratar a la filosofía como mero resultado o preparación (respectivamente) de las ciencias. Las acepciones genitivo-subjetivas, típicas de casi todas las facultades no-filosóficas, se pueden encuadrar aquí (e.g., filosofía del derecho, de la música, de la ciencia, etc., al tomar esos genitivos como si tuvieran una filosofía clarificable en sus propios términos), pero también la idea de filosofía como saber dirigido a alcanzar fines prácticos, ya sean los fines abstractos de la sociedad (e.g., el "filosofismo" definido por Bueno) o del individuo (e.g., "la felicidad").

9. Acepciones parasitarias: la filosofía ni siquiera tendría la capacidad de servir a otros saberes, limitándose a exponer la sustancia de éstos (acaso su historia), a menudo bajo la convicción de estar realizándose así un saber imperfecto, subordinado $(e . g$., las tesis de "la muerte de la filosofía", o las ideas —en ejercicio, casi nunca representadas- que la convierten en doxografía, filología o comentario de textos).

La concepción más extendida en la actualidad (e.g., entre muchos profesores de filosofía) es una mezcla entre las acepciones ancilar y parasitaria, al reducir de dicto la filosofía a su utilidad práctica, concretamente en el sentido "filosofista", que le supone un "carácter cívico" (democrático) y pseudo-antropológico (e.g. "enseñar a pensar"), según la idea de "convivencia pacífica universal mediante el ejercicio del diálogo racional y tolerante" (quedando la filosofía relegada al "reino del discurso" - congresos, periódicos, tertulias, etc.—, según una concepción idealista de la racionalidad). ${ }^{2}$ El modo de lograr este civismo resultaría ser (de facto) una técnica parasitaria: el comentario de textos, lo que revela la ingenuidad de este enfoque.

\footnotetext{
${ }^{2}$ ¿Qué es la filosofía?, pp. 56-9.
} 
Para comprender éstas y otras acepciones, Bueno distingue dos grupos de ideas de filosofía incompatibles, según su forma de entender su conexión con el presente, que hemos de considerar: el primero entiende la filosofía como exenta respecto al presente; el segundo, como implantada en él.

\section{Filosofías exentas: dogmáticas o históricas}

Bueno rechaza con más prolijidad las concepciones exentas, que sólo en el plano de la génesis (histórica o psicológica) son capaces de admitir alguna conexión con su presente (aunque, a veces, ni siquiera admitan eso): "proceden — argumenta - como si, en el plano estructural, la filosofía pre-existiese al presente, como un saber sustantivo hipostasiado, de suerte que pudiera decirse que tal saber llega al presente desde un 'más allá' o de un 'más atrás', para iluminarlo o para oscurecerlo". ${ }^{3}$ Estas acepciones tienden a tratar la filosofía como un saber "de primer grado" y pueden ser de dos tipos:

1. Dogmáticas o escolásticas: pretenden fundarse en principios axiomáticos e intemporales, considerando el contenido filosófico como algo definitivo (e. g., tomismo, cartesianismo, neokantismo, krausismo, Diamat).

2. Históricas o etnológicas: buscan su fundamento en el pretérito o en el presente etnológico. El primer tipo es común en el gremio de profesores de filosofía, que reducen ésta a exégesis, hermenéutica, "interpretación, desvelamiento y retorno incesante a supuestas verdades arcanas que habrían sido ya pronunciadas", ${ }^{4}$ idea esta última reforzada por Heidegger, para quien la hazaña de los presocráticos —el desvelamiento del Ser- habría sido ocultada por la filosofía posterior, o por la idea de una philosophia perennis, acechando siempre el peligro de convertir la filosofía "en filología o incluso en doxografía". ${ }^{5}$ El segundo tipo, correspondiente a las acepciones antropológicas, lo critica Bueno especialmente en Etnología y Utopía, contra la pretensión de la etnología de presentarse como "una alternativa a la filosofía, incluso como su verdugo". ${ }^{6}$

${ }^{3} I b .$, p. 32.

${ }^{4} \mathrm{Ib} ., \mathrm{p} .35$.

${ }^{5} \mathrm{Ib} ., \mathrm{p} .37$.

${ }^{6}$ Bueno, Gustavo. Etnología y Utopía. Respuesta a la pregunta: ¿qué es la Etnología? Serie Antropología, Júcar Universidad, 1987, p. 10. Bueno vuelve al asunto en pp. 136-48. 
Si hay alguna consideración del presente en estas filosofías exentas, se hace siempre desde el pretérito o desde la eternidad.

\section{Filosofías implantadas: adjetivas o críticas}

Las filosofías implantadas, por el contrario, juzgan el pretérito desde el presente. Habría dos tipos:

1. Adjetivas: suponen una implantación radical de la filosofía en el presente, a tenor de la cual ésta se convierte en un saber adjetivo, esto es, en una mera excrecencia del presente, "como un epifenómeno o un pleonasmo" ${ }^{7}$ de su cultura (técnica, científica, literaria, etc.). Suelen ser ideas ancilares o parasitarias, destacando el positivismo, que convierte la filosofía en mera comentadora de las ciencias y consagra la "filosofía espontánea de los científicos", un sucedáneo de la filosofía que, sin más fundamento que el prestigio científico, y con un aparato categorial inadecuado, aborda cuestiones que desbordan su campo de saber ${ }^{8}$ (baste pensar en la Pedagogía o la Cosmogonía física, donde se ocultan tantos "sofistas" del presente). ${ }^{9}$ Cualquier acepción genitivo-subjetiva de la filosofía (no en el sentido objetivo, que Bueno acepta y denomina "filosofía centrada"), ${ }^{10}$ se encuadraría aquí. Un caso radical es La philosophie bantoue (1945) de Placide Tempels.

2. Filosofía crítica: supone una filosofía sistemática de contenido dialéctico, donde se debe presentar "el sistema total de alternativas" filosóficas y justificar la elección de una u otra, no desde la duda o la ignorancia, sino partiendo de saberes previos lo más firmes posible. ${ }^{11}$ La filosofía crítica tiene como uno de sus objetivos la demolición de las filosofías exentas y adjetivas (sin perjuicio de que puedan darse analogías con ellas), ${ }^{12}$ erigiéndose como un "saber totalizador", pero también "democrático" (al negar toda fuente privilegiada de sabiduría). Esta acepción supone "una sustancialidad, al menos actualística, para la filosofía", ${ }^{13}$

\footnotetext{
${ }^{7}$ ¿Qué es la filosofía?, p. 39.

${ }^{8} \mathrm{Ib} ., \mathrm{p} .40$.

${ }^{9}$ Ib., pp. 115-6.

${ }^{10} \mathrm{Ib}$., p. 43.

${ }^{11}$ Ib., pp. 45-6.

${ }^{12} \mathrm{Ib}$., p. 71.

${ }^{13} I b .$, p. 68.
} 
que no es una mera excrecencia de saberes previos, sino un saber "de segundo grado" con su propia materia, las Ideas, que surgen del conflicto entre saberes "de primer grado" (mundanos, técnicos, míticos, políticos, religiosos, científicos, etc.). La filosofía no puede ser un saber definitivo ni una "ciencia del todo", ${ }^{14}$ al estar ligada a su presente, confrontada con otros saberes que le obligan a actualizarse continuamente. Supone también el "dominio" de una "técnica de discusión", ${ }^{15}$ y posee una "misión catártica" "16 respecto a esos saberes que, a partir de los tres ejes del espacio antropológico, Bueno agrupa bajo las etiquetas de Ciencia, Política y Religión (el fundamentalismo científico y democrático, los mitos de la Cultura, la Felicidad, la Izquierda, la Derecha, el ateísmo, etc., ofrecen una imagen clara sobre qué pueda ser esa catarsis). La misión de la filosofía no sería tanto hacer cosas como deshacerlas; en términos platónicos, bajar a la Caverna, triturar ideologías, mitologías y fundamentalismos. El carácter dialéctico y académico de la filosofía se podría resumir así: "pensar es pensar contra otros" (Bueno destacó en 2014 el componente agonístico de la filosofía en su "filosofía del deporte"). ${ }^{17}$ Ésta es la postura de Bueno.

A la escala "sustancialista" en que se plantean tales problemas, acepto la crítica y remito al lector al opúsculo ¿Qué es la filosofía? (1995), pero ahora mantendré que esto no basta para tener una definición completa (que no confundimos con perfecta), como tampoco aparece en El papel de la Filosofía en el conjunto del Saber (1970), su respuesta al ensayo de Manuel Sacristán. ${ }^{18}$ Esta "Filosofía de la Filosofía" o "autofilosofía", ${ }^{19}$ este libro "perifilosófico" cuyo "tema central” sería "el concepto de Filosofía”, tampoco cierra la definición (lo cual jamás significa finalizarla) y, por tanto, no termina de abrir el campo de la filosofía en su verdadera amplitud, acaso por ser "un libro más bien polémico, una respuesta apresurada" al "discurso retórico" de Sacristán. ${ }^{20}$ En estos ensayos tenemos (lo que ya es bastante)

${ }^{14}$ Ib., p. 118.

${ }^{15}$ Ib., p. 115.

${ }^{16}$ Ib., p. 119.

${ }^{17}$ Bueno, Gustavo. Ensayo de una definición filosófica de la Idea de Deporte. Pentalfa, Oviedo, 2014, p. 11.

${ }^{18}$ Sacristán Luzón, Manuel. "Sobre el lugar de la filosofía en los estudios superiores”. Editorial Nova Terra, Barcelona, 1968.

${ }^{19} \mathrm{Ib}$., pp. 61-3.

${ }^{20}$ Bueno, Gustavo. El papel de la filosofía, p. 10. 
una negación de lo que la filosofía no es y una definición del contenido propio de la filosofía, a partir de la distinción entre Conceptos e Ideas. No estoy tan en desacuerdo con lo que Bueno afirma acerca de la filosofía, como con aquello que deja fuera de la definición (sin obviar que, sin duda, la mejor forma de saber qué es la filosofía para Bueno sería atender al resto de sus obras, exposiciones orales, videos, su Escuela, etc., y descifrar allí la filosofía ejercida, más compleja que la representada.)

\section{Filosofía estricta y filosofías oblicuas: un poco de historia}

Para nuestra crítica, hemos de comenzar con la "filosofía en su acepción estricta", esto es, "la filosofía de origen helénico, y más precisamente platónico, académico", con todo su curso posterior en el "área de difusión helénica" ${ }^{21}$ que habría ido sedimentándose en culturas diversas, generando "contenidos universales" (no "de hecho", pero sí "de derecho", por cuanto "el derecho a este saber" no está "contradicho, de un modo solvente, por los derechos reclamados por otros saberes"), ${ }^{22}$ que llegan hasta nuestro presente. Aun aceptando este origen "académico" de la filosofía, la acepción que uno tenga de filosofía determina ya su comprensión de esa filosofía estricta y, por tanto, nuestras razones para comenzar con Platón serán necesariamente distintas (aunque sean complementarias).

Mi crítica, por dejarla formulada desde el principio, es la siguiente: se ha intentado definir, en todo momento, una parte esencial de la filosofía, a saber, su "contenido", y en concreto su contenido propio, en función del cual se entiende su "misión" y su diferencia con otros saberes, pero se ha dejado de lado otra parte esencial, a saber, su "forma". Bueno nos habla de ideas, de saberes, del campo de los saberes, pero echamos en falta al sujeto que idea, sabe, conecta, destruye. Cuando Bueno asegura sentir interés por la acepción exenta de filosofía "en la medida en que ella vaya ligada a una doctrina o conjunto de doctrinas más o menos precisas", delata este interés por el "contenido" del campo filosófico, frente al interés que podría haberse mostrado (también) por la "subjetualidad" de quien hace esa filosofía. Bueno analiza la filosofía, pero olvida al filósofo (aunque éste se encuentre supuesto, tácitamente, pero sólo desde las ideas). ${ }^{23}$

${ }^{21}$ Bueno, Gustavo. ¿Qué es la filosofía?, p. 21.

${ }^{22} \mathrm{Ib}$., p. 107. Sobre la negación del etnocentrismo véase pp. 51-55.

${ }^{23}$ Así ocurre incluso en textos como Bueno, Gustavo. "El concepto de 'implantación de la conciencia filosófica'. Implantación gnóstica e implantación política”. Revista de Occidente. Madrid, 1972, pp. 37-71. 
En su empeño por mostrar que la filosofía es "reflexión" (saber "reflexivo"), debido a la vuelta del saber "de segundo grado" sobre los saberes "de primer grado", y no a la "vuelta del sujeto sobre sí mismo", tiende a alejar al sujeto. ${ }^{24}$ Lo esencial en sus ensayos es mostrar que la filosofía ofrece "contenidos sustantivos", no "adjetivos". ${ }^{25}$ Bueno está preocupado (finis operis) por mostrar (ahora analizaremos por qué) que la filosofía está implantada "social e históricamente", y no en "una supuestamente originaria subjetividad individual de las conciencias humanas", cosa que aceptamos, como también que "el desarrollo de la racionalidad crítica", en las colonias griegas, "no es un proceso individual ('ontogenético'), sino histórico ('filogenético')". ${ }^{26}$ Pero a esto añadimos que esa racionalidad griega también supone un "modo" (actualístico) de subjetualidad (tan histórico como las ideas), propio del filósofo, que no puede quedar neutralizado en la definición de su campo, y que nos obliga a confrontar esa subjetualidad (en la medida en que pueda tener una racionalidad propia, filosófica) que desborda las ideas como sustancialidad de la filosofía.

Bueno se centra ante todo en delimitar el "contenido doctrinal" y el “campo de la filosofía”, que estaría conformado por Ideas. ${ }^{27}$ La razón de este hincapié en el "campo" (aunque éste no sea exento o adjetivo), podría explicarse apelando al contexto crítico de los años $60,{ }^{28}$ cuando Sacristán llegó a proponer el cierre de las facultades de filosofía y la eliminación de sus asignaturas en la enseñanza media, precisamente argumentando que no era una "especialidad". Este contexto es análogo al acaecido en el año 2005 , cuando se organizan varias manifestaciones contra la reducción de la materia en los institutos, repartiéndose en una de ellas cien ejemplares del opúsculo de Bueno, situación que se repite a su manera en el año 2016. ${ }^{29}$

${ }^{24}$ ¿Qué es la filosofía?, p. 98.

${ }^{25} \mathrm{Ib}$., p. 92.

${ }^{26} I b .$, p. 99.

27 “El 'campo de la filosofía' está dado en función de los otros, de sus analogías o de sus contradicciones. Y las líneas más o menos identificables que las analogías o las contradicciones entre las ciencias y otros contenidos de la cultura perfilan, las llamamos Ideas, con el propósito de conferir un minimum de objetivación positiva a los materiales que desencadenan estos procesos tan diversos que englobamos bajo el rótulo de 'filosofía crítica"” (Ib., pp. 46-7).

${ }^{28}$ Así lo admite, e. g., en "Teorema entrevista a Gustavo Bueno", Teorema, vol. III, n 1 (1973): "En El papel de la filosofía, la perspectiva era principalmente esta: ¿Tiene de hecho la Filosofía (en particular, la filosofía académica) un contenido "sustantivo"?” (p. 125).

${ }^{29}$ Véanse las noticias recogidas en http://www.filosofia.org/bol/not/bn045.htm, bajo el título "Estrategias socialdemócratas y lucha pro-filosofía, junio de 2005". (Última consulta: 20/01/2017.) La situación no ha cambiado demasiado a partir de entonces. En el año 2016, 
Este contexto general explica en parte la necesidad de argumentar de manera nítida la existencia de un campo filosófico específico.

A ello se unen los problemas propios de la filosofía moderna en conexión con el auge de las ciencias. La gran difusión de las concepciones oblicuas (giro lingüístico, positivismo lógico, doxografismo de los profesores de filosofía, etc.), casi obligaba a mostrar que lo propio de la filosofía no podía ser abordado por ningún otro saber. Ya en las lecciones de 1929-30 de Ortega y Gasset, editadas bajo el título ¿Qué es filosofía? (inicio de esta tradición — digna de un estudio más prolijo- en España), el madrileño definió "los sesenta postreros años del siglo XIX" como "una edad antifilosófica" ${ }^{30}$ por su repliegue ancilar ante las ciencias (situación que remontaba a Kant), ${ }^{31}$ y no es extraño que también él se esforzara por definir al filósofo como "un especialista, a saber, un especialista en universos". ${ }^{32}$ Este mismo lamento y contraste con la ciencia aparecerá en la Carta sobre el Humanismo (1949) de Martin Heidegger, para quien "hace mucho tiempo, demasiado, que el pensar se encuentra en dique seco", mientras la filosofía "se siente atenazada por el temor a perder su prestigio y valor si no es una ciencia". ${ }^{33}$ Lo mismo ocurre en Was ist Philosophie? (1960) de Dietrich von Hildebrand, donde las comparaciones entre ciencia y filosofía son continuas, ${ }^{34}$ culpando al positivismo del desinterés hacia la filosofía, que él pretende "rehabilitar" 35 argumentando que el "objetivo principal" de la filosofía es "descubrir estados de cosas a priori, no empíricos", ${ }^{36}$ en virtud de los cuales - tras una serie de rodeos teológicos- las ciencias serían hijas de una específica "sed filosófica". ${ }^{37}$ El propio Bueno, en sus Ensayos materialistas (1972), definió su proyecto filosófico como producto de un "ideal hegeliano" que

el propio Bueno decidió republicar en El Catoblepas el prólogo de 1968 a El papel de la filosofía, respondiendo a lo que denominó "la crisis de la filosofía administrada en España". Véase Bueno, Gustavo. "Prólogo a El papel de la filosofía en el conjunto del saber", El Catoblepas, $\mathrm{n}^{\circ} 173$ (2016), p. 2.

${ }^{30}$ Ortega y Gasset, José. ¿Qué es filosofía? Ediciones de la Revista de Occidente, Madrid, 1976, p. 33 .

${ }^{31} \mathrm{Ib}$., p. 48. Cf., p. 89.

${ }^{32}$ Ib., p. 110.

${ }^{33}$ Heidegger, Martin. Carta sobre el Humanismo, trad. de Helena Cortés y Arturo Leyte. Alianza, Madrid, 2006, pp. 13-4.

34 von Hildebrand, Dietrich. ¿Qué es la filosofía? [1973], trad. de Araceli Herrera. Encuentro, Madrid, 2000, véase toda la "Introducción" (pp. 9-17). Cf., e. g., p. 183, p. 187, p. 202.
${ }^{35} \mathrm{Ib}$., p. 16.
${ }^{36} \mathrm{Ib}$., p. 66.
${ }^{37} I b .$, p. 218. 
consistía en "colaborar a que la Filosofía no sea sólo amor a la sabiduría, sino sabiduría académica". ${ }^{38}$ Este esquema se repite a lo largo del siglo, llegando hasta Qu'est-ce que la philosophie? (1991) de Gilles Deleuze y Félix Guattari, para quienes "el filósofo es un especialista en conceptos" ${ }^{39}$ y la filosofía es "la disciplina que consiste en crear conceptos", ${ }^{40}$ estatuto que otras disciplinas (Sociología, Lingüística, Psicoanálisis, etc.), pero también la publicidad o la mercadotecnia, le estarían disputando. ${ }^{41}$ En un contexto más amplio (del cual este momento crítico sería sólo un síntoma), la crítica kantiana, marxista, nietzscheana y heideggeriana a la metafísica, convertida en ejercicio trascendental replegado ante las ciencias, en ideología social, en impotencia estética u ocultamiento del Ser, podrían explicar la necesidad de una noción de filosofía como garante de un saber especializado.

Para lograr esa defensa de la filosofía, las concepciones subjetualistas parecían especialmente problemáticas. Los primeros libros veintescos de esta temática, como La esencia de la Filosofía (1907) de Dilthey, ${ }^{42}$ o los ensayos de "filosofía de la filosofía" de José Gaos, ${ }^{43}$ acababan ofreciendo una definición ancilar, donde predominaba una idea de filosofía como cosmovisión (epocal o personal), acorde con la idea fichteana, según la cual el tipo de filosofía que uno elige depende del tipo de hombre que se es. ${ }^{44}$ No menos problemático fue el etnologismo posterior (e. g., la roussoniana "identificación entre la Humanidad y el Salvajismo" de Levi-Strauss, que recaía en posiciones gnósticas). ${ }^{45}$ En 1956, Heidegger publicaba Was ist das —die Philosophie?, definiendo la filosofía como "la verdadera administradora de la razón", ${ }^{46}$ una definición idealista por mucho que se vinculara al "mundo griego" 47 (en el que habría nacido el propio modo de preguntar implicado en la pregunta, ${ }^{48}$ y desde el cual se pretendía entender la "era atómica", ${ }^{49}$

${ }^{38}$ Bueno, Gustavo. Ensayos materialistas. Taurus, Madrid, 1972, p. 16.

${ }^{39}$ Deleuze, Gilles \& Guattari, Félix. ¿Qué es la filosofía?, trad. de Thomas Kauf. Anagrama, Barcelona, 1997, p. 9.

${ }^{40} \mathrm{Ib} ., \mathrm{p} .11$.

${ }^{41} \mathrm{Ib} ., \mathrm{p} .16$.

${ }^{42}$ Dilthey, Wilhelm. Esencia de la filosofía, trad. de Elsa Tabernig. Losada, 2003.

${ }^{43}$ Gaos, José. Filosofía de la filosofía. FCE, Madrid, 2009.

44 "Was für eine Philosophie man wähle, hängt sonach davon ab, was man für ein Mensch ist” (Fichte, Johann Gottlieb. Versuch einer neuen Darstellung der Wissenschaftslehre. Felix Meiner Verlag, Hamburg, 1984, p. 17).

${ }^{45}$ Bueno, Gustavo. Etnología y Utopía, p. 142. Cf., p. 95 y p. 140.

${ }^{46}$ Heidegger, Martin. ¿Qué es la filosofía?, trad. de Jesús Adrián Escudero. Herder, Barcelona, 2004, p. 31.

${ }^{47} \mathrm{Ib} ., \mathrm{p} .34$.

${ }^{48}$ Ib., p. 39.

${ }^{49}$ Ib., p. 37. 
según una acepción exento-histórica de filosofía, de tipo ancilar, con ocasionales ribetes dogmáticos). Sus hilos conductores llegan hasta el presente, considerado por algunos, acentuando el lamento de Hildebrand, como "el momento de la historia de nuestra cultura en el que la filosofía ha devenido objeto de mayor indiferencia", definiéndola como "la actitud y la disposición que hace posible la ciencia y el saber propiamente tal", esto es, como "conditio sine qua non de la verdad", en la órbita de Kant y Heidegger. ${ }^{50}$ La Filosofía de la filosofía (2010) del Proyecto Enciclopedia Iberoamericana de Filosofía supone, por su parte, una prueba más de ancilaridad, al ejercer pese al título- una filosofía parasitaria, limitada a comentar las acepciones de otros autores. ${ }^{51}$

Las acepciones oblicuas, unidas a las subjetualistas, suponían entonces, y aún hoy, neutralizar el contenido propio de la filosofía, mientras las concepciones no-subjetuales (aitiológicas, gnósticas, etc.), apenas gozaban de epígonos (salvo en los casos, ya marginales en el año 1995, del neotomismo y el Diamat). Una tradición tan amplia como esta podría explicar la crítica bueniana al existencialismo, el idealismo, la antropología, la sociología, etc., pero también podría aclarar por qué, en su confrontación con las ciencias, ha descuidado los componentes que llamaremos "subjetuales" o "formales", poniendo el acento en asuntos "objetuales" o de "contenido". Su preocupación por "el papel de la filosofía en el conjunto del saber" y "el lugar de la filosofía en la educación" (según los subtítulos respectivos de ambas obras), concebidos en ambos casos en contraste con el papel y el lugar de la ciencia, parecen haberle obligado a analizar la filosofía desde el prisma científico, y así como las ciencias neutralizan —según la teoría del cierre categorial - al sujeto operatorio, la definición de Bueno habría neutralizado (finis operis) la modalidad subjetual de la filosofía.

En un contexto crítico no menos acentuado, ni menos cientificista, proponemos analizar esa modalidad, considerando que la definición de la filosofía ha de hacerse sin concesiones a perspectivas parciales, como la proyectada desde la idea de ciencia, necesariamente capciosa a la hora de comprender la filosofía. Nadie podrá objetar que estamos ante otra pregunta, que no sería tanto “¿qué es la filosofía?" como "¿qué es un filósofo?”, pues ambas son las dos caras de la misma moneda. Tampoco se objetará que entramos con ello en el farragoso terreno del hombre universal; una

${ }^{50}$ Fernández Liria, Pedro. ¿Qué es filosofía?: Prólogo a veintiséis siglos de historia. Akal, Madrid, 2010, pp. 7-12.

${ }^{51}$ Nudler, Oscar (ed.). Filosofía de la filosofía. Trotta, Madrid, 2010.

52 Bueno, Gustavo. ¿Qué es la filosofía?, p. 68. 
respuesta así sería tan burda como objetar a los opúsculos de Bueno entrar en el farragoso terreno de los saberes universales; damos por descontado que la subjetualidad filosófica, con su primera plasmación escrita en los diálogos platónicos, también está implantada en su presente histórico y puede tener un carácter crítico.

\section{Filosofía reflexiva e imperativa}

Para esbozar una definición lo más completa posible, propongo una distinción básica en dos partes indesligables, pero analizables por separado, correspondientes a dos momentos necesarios para definir la filosofía y que se retroalimentan. Entre ellos se daría una tensión dialéctica que cada filósofo resuelve (continuamente) a su manera:

1. El primero estaría conformado por "cuestiones de contenido": trataría la "sustancialidad" de la filosofía definida por Bueno ${ }^{52}$ (una sustancialidad "actualística", no identificable con una "sustancia exenta"), ${ }^{53}$ en virtud de la cual se pretende realizar una "Geometría de las Ideas" 54 (una denominación crítica, comprendida como "norma regulativa", que no presupone la posibilidad de su construcción efectiva), ${ }^{55}$ definiéndose la filosofía como una "especialidad". ${ }^{56}$

2. El segundo (en confrontación con el uso de expresiones como "sustancialidad" y "contenido"), podemos llamarlo, según la lógica de los conceptos conjugados, "cuestiones de forma", vinculadas al hecho de filosofar — que se deriva, además, de la propia acepción reflexivo-crítica, al resultar obvio que no existe la filosofía, sino más bien el filosofar - y, por tanto, a los filósofos. Esta sería la "modalidad" de la filosofía, en referencia al "modo" subjetual del filósofo, es decir, su forma o modo de ser, que entenderemos también en un sentido implantado-crítico.

Si, para comprender las "cuestiones de contenido", podemos admitir prima facie la definición bueniana (aunque, como veremos, tendremos que redefinirla en un contexto distinto y más amplio), sobre las "cuestiones de forma" habría mucho que añadir. Llamaremos a la definición de Bueno el

${ }^{53} \mathrm{Ib} ., \mathrm{p} .64$.

${ }^{54}$ Ib.: El papel de la filosofía, p. 17.

${ }^{55}$ Ib.: ¿Qué es la ciencia? La respuesta de la teoría del cierre categorial. Ciencia y Filosofía, Pentalfa, Oviedo, 1995, p. 112.

${ }^{56}$ Véase "La filosofía como especialidad académica", El papel de la filosofía, pp. 243 y ss. 
"momento reflexivo" de la definición, y a éste sumaremos, en lo que sigue, un "momento imperativo". La "definición imperativa" amplía el "campo" de la filosofía sin destruir el abierto por el momento reflexivo, al que incluye y redefine a otra escala.

La definición reflexiva presenta la filosofía como un saber de segundo grado, con un campo sobre el cual ejerce su crítica; la definición imperativa, como una actividad (que comporta una actitud, un modo de ser) ejercida por filósofos, cuya modalidad estaría definida críticamente a partir de un imperativo básico: dedicarse exclusivamente a lo mejor, es decir, a las cuestiones más importantes, sean éstas cuales fueran, en virtud de unas determinaciones subjetuales (institucionales, críticas) que obligan a reconocer los límites ontológicos del sujeto, en confrontación con modos de subjetualidad no-filosóficos o que niegan esas determinaciones. La reflexividad supone que, cualquiera que sea el material ideal que uno aborde críticamente, tendrá como resultado una filosofía (mejor o peor); la imperatividad, que, sea cual sea el campo al que se dedique el filósofo, éste podrá ser filosófico si responde al imperativo filosófico de quien lo ejerce (mejor o peor).

Es así como el momento modal de la definición redefine al momento sustancial, retraduciéndolo en estos términos: la sustancialidad de la filosofía no se puede definir como el campo único de la filosofía, que la convertiría en una especialidad, sino más bien como su campo propio, que en este sentido huye (críticamente) de la especialización. Si consideramos, e. g., la obra de Aristóteles, el momento sustancial de la definición nos haría considerar la Teología (como metafísica de la Idea de Dios) como un apartado del campo filosófico, quedando la Física (en lo que tuviera de conceptual) como un saber de primer grado (no-filosófico). El momento modal, sin embargo, nos haría considerar la Física como una parte esencial de la Filosofía (como, de hecho, la consideraba el propio Aristóteles), no en virtud de su contenido "ideal" (aunque pueda tenerlo), ni como algo meramente instrumental (para-filosófico), sino en virtud de su importancia "filosófica".

Uno no sólo es filósofo por tratar temas filosóficos ("el territorio de las ideas", frente al "territorio de los conceptos"), ${ }^{57}$ sino también por el modo de tratarlos, esto es, por ser un filósofo. Bueno se limita a definir una parte del campo filosófico, a saber, lo propio de sus contenidos, pero lo propio no es necesariamente lo esencial, ni el contenido es la única parte de la definición. La definición reflexiva supone que la filosofía son las ideas; la

57 Véase Bueno, Gustavo. "En torno a la distinción entre 'Conceptos' e 'Ideas'”. El Catoblepas, no 127 (2012), p. 2. 
imperativa, que son las cuestiones más importantes, sean ideas, conceptos, técnicas o formas de ser. Según el momento reflexivo, la filosofía es un saber de segundo grado; según el imperativo, es una actividad (el filosofar) desarrollada por filósofos, siguiendo la máxima de dedicarse exclusivamente a lo mejor. Eso incluye necesariamente saberes de segundo grado, pero también saberes de primer grado, actitudes, modos de ser.

A esta distinción subyace una tradición - ya asumida por Platón y su Idea del Bien- según la cual lo más importante que puede enseñar la filosofía es (algo que tomaremos en un sentido crítico) a hacer el Bien, en el sentido de buscar siempre hacer (conocer, realizar, criticar, etc.) lo mejor. Esto no se puede confundir con la "geometría de las ideas", pues sería como creer que la filosofía sólo apunta hacia sí misma, incluso cuando apunta a otros saberes, de los que meramente se nutriría para coger fuerzas y seguir a lo suyo: como si su geometrizar tuviera interés por sí mismo y no por lo que enseña sobre la vida y sobre las cosas (donde resulta un sinsentido afirmar que éstas son "filosóficas" o "no-filosóficas”). Así lo define

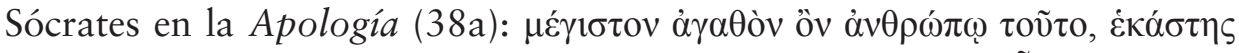

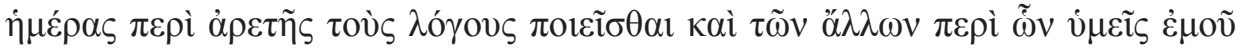

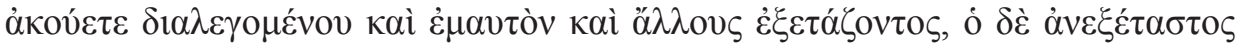

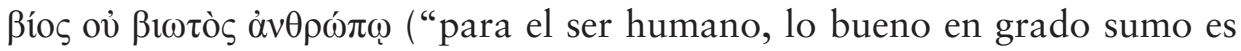
concebir cada día razones sobre la virtud y sobre el resto de cosas de las que me habéis escuchado dialogar, ya sea examinándome a mí mismo o a los demás, porque una vida sin examen no es una vida humana”), después de haber criticado al resto de oficios por creerse sabios acerca de $\tau \grave{\alpha} \mu \mu^{\prime} \gamma 1 \sigma \tau \alpha$,

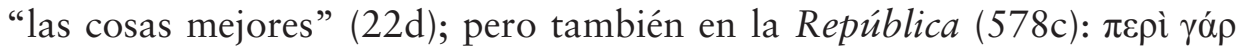

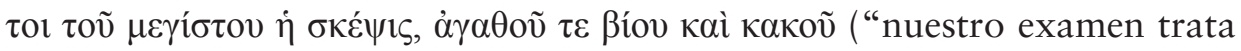
sobre lo más importante: el modo de vivir bien o mal"). No es casualidad que casi toda la obra de Platón esté dedicada a exponer el modo de ser de una subjetualidad concreta: Sócrates.

Este componente se puede encontrar en otros momentos fundamentales de la tradición griega. Pablo de Tarso, en su primera carta a los Tesalonicenses, propone varios imperativos, todos ellos dirigidos a la vida cristiana, que considera el mejor modo de vida posible. Uno de ellos (5:21)

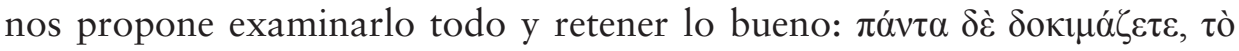
$\kappa \alpha \lambda o ̀ v ~ \kappa \alpha \tau \varepsilon ́ \chi \varepsilon \tau \varepsilon$, que la Vulgata traduce como "Omnia autem probate: quod bonum est tenete". Lo bello y lo bueno se identifican en la traducción, que en el fondo significan "lo mejor", como refleja el ideal griego de la

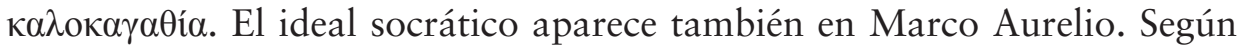

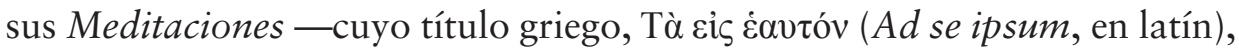


muestra también ese componente subjetual—, cuando le preguntaron cuál era su profesión, respondió: "Ser bueno" [ảy $\alpha \theta$ òv Eĩval]. La amplitud del concepto de ảy $\alpha \theta$ ó $\varsigma$ es evidente tanto en Platón como en Marco Aurelio: el emperador aclara explícitamente que "ser bueno" supone dedicarse a cuestiones humanas y físicas. ${ }^{58}$ Igual que las ideas cambian históricamente y surgen del conflicto de múltiples saberes, también lo hacen los filósofos y, por tanto, su "bondad". Este concepto, críticamente entendido, implica que uno es filósofo todo el tiempo, no sólo cuando se ocupa de ideas, definiendo algo específico de la filosofía frente a otras actividades: mientras el político, el científico, etc., sólo lo son mientras se ocupan de su oficio, el filósofo lo es siempre, pues su "oficio" y su "vida" tienden a identificarse. Una buena parte del filosofar socrático, como su modo de comportamiento ante el tribunal (pero también su modo de vestir, del que tanto hablan sus discípulos), queda fuera de la definición reflexiva, pero es fácilmente asimilable desde la imperativa.

Toda definición de filosofía ha de tener en cuenta las ideas, pero también a los filósofos y su modo (crítico) de ser, y por tanto al resto de saberes, no sólo porque lo propio de la filosofía nos obligue a comenzar por saberes de primer grado, para alcanzar gradualmente otros de segundo grado, sino también porque aquéllos nos interesan (filosóficamente) qua tales. Aunque requiera múltiples especializaciones, el filósofo se distingue del científico, precisamente, por no recluirse en una especialidad.

Las numerosas apologías de la filosofía son una prueba de cómo la filosofía (de un modo esencial) son "los filósofos", y no sólo "las ideas". La tradición filosófica no sólo hereda libros, tratados e ideas, sino también modos de ser. Desde la Apología de Sócrates, que es también la de Platón (todos sus diálogos están teñidos de una apología de la filosofía), tenemos los casos de Aristóteles (que, según la doxografía, abandonó Atenas para evitar otro ataque contra la filosofía), y de manera evidente en los estoicos, cínicos o epicúreos. Una historia del modo de vida filosófico habría de servir para aclarar la definición (aunque lo esencial para la filosofía, según lo

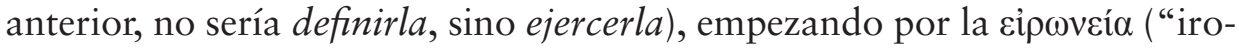
nía”) socrática, acaso el modo esencial del filosofar, que implica un modo de alejamiento de uno mismo, de su propia reflexividad institucional -el

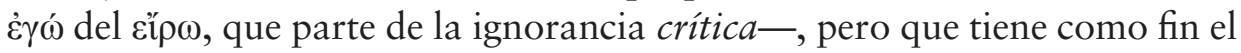

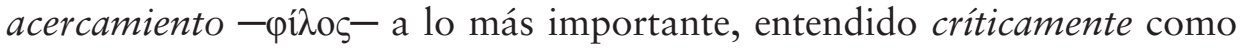

${ }^{58}$ Marcus Aurelius. Ad Se Ipsum, ed. de Jan Hendrik Leopold. B. G. Teubneri, Leipzig, 1908, Libro IX, 5. 


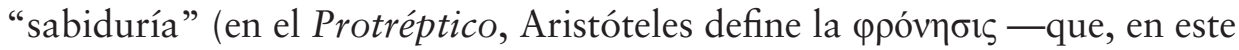
texto, equivale a бopía- como "el más importante de los bienes", ${ }^{59}$ lo que explicaría ad hoc que ese modo subjetual crítico se defina como "filosofía” y no de otro modo). La determinación mostrada ante la muerte o en situaciones adversas (e. g., por Sócrates, Cicerón, Séneca, Agustín, Boecio, Moro, Bruno, Spinoza), apunta en esta dirección, que justificaría la denominación de vidas o muertes filosóficas. Los diálogos platónicos hablan por sí solos, pero la doxografía está plagada de ejemplos. El caso del eléata Zenón, que habría sido arrestado por un tirano tras conspirar contra él, accediendo a revelarle al oído el nombre de sus colegas para, a continuación, arrancarle la oreja de un mordisco, nos ofrece una imagen de la dialéctica - atribuida a Zenón- más luminosa que la ofrecida en los manuales ${ }^{60}$ (situación que hemos vuelto a observar con la muerte de Bueno). ${ }^{61}$

En De revolutionibus afirma Copérnico que, entre las principales investigaciones, "han de abarcarse y seguirse con el mayor afán las que versan sobre las cosas más bellas y más dignas del saber", entre las cuales se encontrarían "las maravillosas revoluciones del mundo". ${ }^{62}$ Al hablar así, Copérnico está haciendo filosofía. Si su afirmación es cierta, la astronomía matemática sería filosofía, por mucho que la filosofía sea mucho más. (Esto es así incluso históricamente, como muestra el caso de Galileo, que a partir de esta convicción trató de presentarse como filósofo, no sólo como matemático. $)^{63}$ Lo esencial de la filosofía no es su "especialidad", sino su modo crítico-imperativo de ser, que incluye, pero desborda, toda especialización. La filosofía no es sólo física, pero es también física. La idea de los saberes mundanos o científicos como rodeo hacia la filosofía, desde la acepción imperativa, sólo parecería justificable tras suponer un componente metafísico de corte neoplatónico, propio de una definición exenta y no-subjetual, donde el saber segundo-genérico —ordo cognoscendi- pudiera coincidir con la forma de una totalidad que —ordo essendi- estaría constituida en su expresión esencial y suprema por ideas.

\footnotetext{
${ }^{59}$ Aristóteles. Protréptico, ed. de Carlos Megino Rodríguez. Abada, Madrid, 2006, fr. 40.

${ }^{60}$ Diogenes Laertius: Op. cit., VII, 1.

${ }^{61}$ Véanse las decenas de necrológicas recogidas aquí: http://www.filosofia.org/bol/not/ bn071.htm

${ }^{62}$ Copérnico, Nicolás. Sobre las revoluciones (de los orbes celestes), ed. de Carlos Minguez Pérez. Tecnos, Madrid, 2009, p. 13.

${ }^{63}$ Véase, e. g., Mario Biagioli: Galileo cortesano, trad. de María Victoria Rodil, Katz, Madrid, 2008, p. 277.
} 
Im-portante (de “in-portare”) se opone a ex-portante. Algo es importante porque nos parece digno de llevar, tener, mantener, acrecentar, frente a aquello que consideramos alejable, rechazable, superfluo (sin perjuicio de que lo mejor requiera a menudo el paso por lo menos bueno). Esto supone un sujeto que acerca, mantiene, en un sentido no sólo ideal, sino también mundano: mantener es, etimológicamente, tener en la mano ("manutenere"), y tener implica un sujeto que se ve impelido ("tenetur") a hacer algo, incluyendo lo que uno tiene que hacer frente a otras cosas, llegando (como posibilidad crítica) a lo importante en grado superlativo: "el Bien", "lo mejor", que no sólo ha de serlo, según el principio de Symploké, una multiplicidad de cosas, sino también cada una de ellas en muchos sentidos: no sólo en sentido ético o psicológico, sino también estético, gnoseológico, ontológico, etc., en disputa continua. Igual que no podemos incluir en la definición de filosofía ideas concretas, debido a su actualismo y continua oposición, tampoco podemos definir qué es lo importante, salvo en el ejercicio mismo del filosofar de un presente dado. Cómo se conciba esta importancia (siempre plural) sólo puede ser definido negativamente, de ahí que nunca tengamos una definición perfecta.

\section{Filosofías imperativas exentas e implantadas}

Igual que las ideas de filosofía, según su contenido, se pueden agrupar en exentas o implantadas, se puede hacer lo mismo según su forma:

\section{Filosofías exentas:}

1. Dogmáticas: las cuestiones más importantes son eternas (e. $g$., "filosofía primera" como "teología").

2. Históricas o etnológicas: las cuestiones más importantes son las legadas por la tradición (e. g., "el mayor de los asombros" con que Heidegger denota un supuesto interés helénico por "el ente en el ser" ${ }^{64}$ de modo que el "camino hacia la respuesta" 65 sobre la filosofía consistiría en "destruir" la historia filosófica para "abrir nuestros oídos, liberarlos a lo que en la tradición se nos transmite como ser del ente") ${ }^{66}$ o por el presente etnológico (lo importante como resultado de la curiosidad "humana").

${ }^{64}$ Heidegger, Martin. ¿Qué es la filosofía?, p. 44. Luego dirá que el páthos del asombro (thaumázein) es el arché de la filosofía (p. 58).

${ }^{65} \mathrm{Ib} ., \mathrm{p} .54$.

${ }^{66} \mathrm{Ib}$., p. 55. Aunque luego hable de "escuchar la voz del ser" (p. 63) para referirse al presente, este presente está ya visto desde el pasado (y no al revés), y por tanto sigue siendo 


\section{Filosofías implantadas:}

3. Adjetivas: las cuestiones más importantes surgen de ciertas instituciones no-filosóficas (e. g., teología, ciencia, etc.).

4. Críticas: las cuestiones más importantes se deciden en cada presente, incluso contra ese presente ${ }^{67}$ y (como posibilidad) contra la filosofía en sentido propio, anclada en una subjetualidad concreta. La confrontación de saberes obliga a tratar con ideas, pero no se descarta criticarlas por medio de conceptos, técnicas, etc., sin que uno deje de ser filósofo por operar así. Actuar filosóficamente es tratar ideas, pero también negarse a hacerlo; no sólo criticar otros saberes y técnicas, sino también ejercerlos. La filosofía crítica puede destruir las pretensiones de especialización de la Geometría de las Ideas, asumiendo que, en un momento dado, hay que actuar como científico, técnico, político, etc., siendo ésta una decisión filosófica. Como ilustra el caso de Marco Aurelio, la filosofía puede tener incluso su momento militar. El filósofo supone un saber crítico, pero también un modo de ser crítico, opuesto a otros modos de ser: por eso el filósofo se compara a menudo con el (que es sólo) político, científico, artesano, poeta, etc. (e. g., en los diálogos de Platón).

Esta definición permite contemplar la racionalidad de las definiciones clásicas de filosofía, subjetuales o no. Las acepciones aitiológicas, gnósticas o existencialistas, por ejemplo, denotan una racionalidad filosófica en la medida en que, de existir sus referentes (causas primeras, etc.), éstos tendrían gran interés filosófico, con independencia de que esos referentes fueran o no "ideales". Desde una perspectiva subjetual, sería irracional que uno se negara, en virtud de una definición de filosofía dada, a tratar con primeras causas sólo porque fueran (supongamos) "conceptuales”. Lo mismo

una acepción exenta. Sus referencias finales a la "poesía”, por otra parte, que podrían tener un componente ancilar, nos acercarían a una acepción dogmática, aunque Heidegger es tan oscuro que resulta imposible saberlo.

${ }^{67}$ Es fácil comprobar en la historia de la filosofía cómo los filósofos se han esforzado a menudo en mostrar que las cosas consideradas importantes por la mayoría — no tanto en el sentido del "vulgo", como en el sentido de "la totalidad de los oficios" (poetas, políticos, científicos, técnicos, etc.) — no lo eran, pero también lo contrario: cómo la mayoría de oficios, cuando ha opinado sobre los filósofos, ha considerado que se dedicaban a cosas de poca monta. Una historia de casos sobre esta confrontación, que abundan desde el nacimiento de la filosofía (Tales y la sierva tracia, Epícrates y las calabazas, etc.), sería reveladora. 
ocurriría a pequeña escala: los saberes aportados por la biología, la música, etc., pueden tener interés propio para el filósofo, contengan o no ideas.

La acepción psicologista (filosofía como amor a la sabiduría) resulta también más racional que antes: el filósofo quiere saber, tiende a la sabiduría, pues ésta no excluye, en principio, nada, entendida como un ideal crítico de saber y de sabio: el filósofo quiere saber lo más importante y hacer lo mejor. La idea crítica de sabiduría está presupuesta en el añadido "amor": algo que se quiere, se busca, pero aún no se tiene, y se considera valioso en grado sumo. Lo más importante incluye conceptos, ideas, pero también cosas, técnicas, subjetualidades: öv $\theta \rho \omega \pi \mathrm{ov} \zeta \eta \tau \tilde{\omega}$ — hominem quaero, en latín-, es una expresión clave en la historia de la filosofía, atribuida al cínico Diógenes. ${ }^{68}$ No hay más abstracción en sostener que la filosofía se ocupa de ideas (sin definir cuales), que en sostener que el filósofo ama la sabiduría (sin concretar cuál). Las acepciones subjetuales (según el imperativo crítico), aciertan al introducir esa modalidad: ha de haber "amor" porque hay "sujeto", y más precisamente porque ese sujeto está atravesado por otros sujetos y objetos que le constituyen, como está atravesado por su presente y sus limitaciones subjetuales (fisiológicas, familiares, laborales, etc.). La filosofía no se puede plantear como un oficio de "ideas", como si las capacidades subjetuales fueran ilimitadas, pues los sujetos están limitados, no sólo por las ideas, sino también por su propia existencia.

De ahí proviene la racionalidad del existencialismo, al considerar importante asimilar la idea de subjetualidad — como la encontramos, por ejemplo, en Unamuno y Ortega ("la vida")— para definir la filosofía (idea que redefine Bueno en su filosofía del Ego trascendental y que, paradójicamente, no tiene en cuenta al definir la filosofía). ${ }^{69}$ Unamuno tiene buenas razones para afirmar que "el hombre concreto, de carne y hueso, es el sujeto y el supremo objeto a la vez de toda filosofía, quiéranlo o no ciertos sedicentes filósofos", y que "lo que en un filósofo nos debe más importar es el hombre", concluyendo en primera persona: "Me dicen que he venido a realizar no sé qué fin social; pero yo siento que yo, lo mismo que cada uno de mis hermanos, he venido a realizarme, a vivir". Si ese "fin social" fuera la Geometría de las Ideas, podríamos afirmar con Unamuno que la filosofía se convierte en "obra de especialización diferenciada" y tiende a ser "filosofería, erudición pseudo-filosófica”, haciendo del filósofo "un pedante, es

${ }^{68}$ Diogenes Laertius: Lives of Eminent Philosophers, ed. de R. D. Hicks [1925], Harvard University Press, Cambridge, 1972, VI, 2.

${ }^{69}$ Véase Bueno, Gustavo. El Ego trascendental. Pentalfa, Oviedo, 2016. 
decir, un remedo de hombre". ${ }^{70}$ No muy lejos de esta idea se encontraba, en el fondo, el propio Sacristán, cuando sostenía que "el tipo institucional del licenciado en filosofía no sólo no merece el nombre de filósofo, sino que es incluso una cómica degeneración de ese programa de conducta". ${ }^{71}$

Ortega, proponiendo ahondar en "nuestra propia existencia" y tras asumir que "las verdades fundamentales tienen que estar siempre a la mano", ${ }^{72}$ enumera algunos ingredientes de la vida (reconocibles antes o después) como el "saberse existiendo", ${ }^{73}$ el "encontrarse en un mundo", ${ }^{74}$ su "carácter súbito e imprevisto" ${ }^{75}$ el "sentirnos forzados a decidir lo que vamos a ser" ${ }^{76}$ o el paradójico ser "lo que aún no es" (o sea, el anticipar-se al "futuro"), ${ }^{77}$ que utiliza para responder al "problema primero filosófico", a saber, determinar "la realidad radical e indubitable", que no sería la "conciencia" o "el sujeto" sino "la vida, que incluye, además del sujeto, el mundo". Obviamente, ésta no puede ser "la filosofía", sino sólo "su capítulo preliminar", ${ }^{78}$ pues cada vida es distinta y la filosofía ha de ser una "forma particular del vivir", ${ }^{79}$ con su propia jerarquía de intereses, posibilidades, etc., pero sí es útil para acometer la definición (siempre crítica y negativa) del filosofar. Ortega reconoce que "la doctrina filosófica, eso que está o puede estar en libros, es sólo la abstracción de la auténtica realidad 'filosofía' —es sólo su precipitado y su cuerpo semimuerto", ${ }^{80}$ pues lo esencial - y esto es lo más cercano al momento imperativo- es "vivir en plena forma" ${ }^{81}$ ¿Para qué íbamos si no a tratar con Ideas? Aunque Ortega no define ese vivir filosófico, salvo con la respuesta parcial que hemos visto al principio, acierta al asumir este componente subjetual. "Vivir en plena forma" parece una forma de decir "dedicarse a las cuestiones más importantes" o "hacer lo mejor", como lo era "ser bueno" en Platón y Marco Aurelio.

Esta modalidad no se agota en las Ideas (las “doctrinas” de Ortega), pues requiere comprender esas condiciones subjetuales asimiladas crítica-

${ }^{70}$ Unamuno, Miguel. Del sentimiento trágico de la vida, en sus Obras completas, X, ed. de Ricardo Senabre. Biblioteca Castro, Madrid, 2009, pp. 275-86.

${ }^{71}$ Sacristán Luzón, Manuel. "Sobre el lugar de la filosofía en los estudios superiores”, p. 18.

72 Ortega y Gasset, José. ¿Qué es filosofía?, p. 224.

${ }^{73} \mathrm{Ib}$., p. 226.

${ }^{74}$ Ib., p. 228.

${ }^{75}$ Ib., p. 231.

${ }^{76}$ Ib., p. 233.

77 Ib., p. 235.

${ }^{78}$ Ib., p. 239.

${ }^{79} \mathrm{Ib}$., p. 241.

${ }^{80}$ Ib., p. 250.

${ }^{81} \mathrm{Ib}$., p. 260. 
mente. Los filósofos se oponen necesariamente a "dejar que las costumbres, los prejuicios, los usos, los tópicos se instalen en su interior, los hagan vivir a ellos y tomen sobre sí la tarea de hacerlos vivir", ${ }^{82}$ y eso implica reconocer el papel esencial de lo subjetual en la definición de filosofía. El filósofo no puede estar jamás en el "taller" especializado de las ideas, sino en el "mundo" (histórico, con-vivido, etc.), como un aficionado (críticamente) de todo, que aspira a saber (saborear, hacer) las cosas más importantes, sean cuales fueran, sin poder negar de antemano su interés por nada, sea "ideal" o no, porque su vida es limitada (es mundo, historia, instituciones, etc., sin identificarse jamás con un "oficio" —eso sería sociologismo-, o con una subjetualidad "humana" —etnologismo-); la filosofía implica vivir (no sólo trabajar - con Ideas o con martillos-) críticamente (con Ideas, pero quizá también con martillos).

Esto encaja con las involucraciones históricas entre ideas y conceptos. La idea de filosofía como "madre" de las ciencias es tan plausible como la idea de filosofía como "hija" de ellas. ${ }^{83}$ Lo atestigua la importancia de los estudios prebiológicos de Aristóteles sobre los animales para el desarrollo de la Biología; de la idea del alma agustiniana para el desarrollo de la Psicología; de la filosofía neoplatónica, para el desarrollo de la Física matemática; de la crítica marxista de la Sociedad, para el desarrollo de la Sociología, etc. Esta involucración se da a menudo en un único sujeto: el Discurso del método (cuyo inicio subjetual no hace falta recordar) es una introducción a tres obras consideradas hoy científicas, pero también podríamos verlo al revés: su filosofía es su Dióptrica, Meteoros y Geometría, que sirven de preparación al Discurso.

También las definiciones ancilares y parasitarias tienen un sesgo filosófico que la definición re-flexiva no puede explicar, salvo de forma historicista. Puede ser importante para el filósofo entregarse plenamente a otros saberes, incluso puede ser una necesidad, como sugiere Ortega en las citadas lecciones, al argumentar que en aquella edad "anti-filosófica", positivista, las cosas fueron "como tenían que ser", aceptando además que "no pocas calidades de la nueva filosofía son debidas a aquella etapa de forzada

\footnotetext{
${ }^{82}$ Ortega y Gasset, José. ¿Qué es filosofía?, pp. 262-3.

83 Véase Bueno, Gustavo. "Respuesta, en 1978, a la pregunta ¿Qué es el cierre categorial?". El Catoblepas, n ${ }^{\circ} 108,(2011):$ "Más próximo a la realidad sería decir que son las ciencias las 'madres' de la filosofía - pero tampoco sería de todo punto exacto. La filosofía procede de otras fuentes, principalmente de los grandes mitos neolíticos [...]. Lo que ocurre es que la constitución de las ciencias [...] marca profundamente a la filosofía y la imprime una dirección característica” (p. 2).
} 
humildad", ${ }^{84}$ entre las cuales podemos incluir nosotros (como reacción) la crítica bueniana al cientificismo, pero también el hecho de que Bueno defina la filosofía, sobre todo, por oposición a las ciencias, como también hace Ortega ${ }^{85}$ (para quien ya "la ciencia misma, como totalidad, es un mito"). ${ }^{86} \mathrm{El}$ mayor reproche que un filósofo puede hacerle a un científico sería el de no estar dedicándose a las cuestiones más importantes (aunque eventualmente pueda contribuir a ellas), pues su bondad está delimitada esencialmente por su oficio, y no por su búsqueda crítica, sin aspirar (subjetualmente) a comprender dialécticamente las diversas alternativas. Y esto puede hacerse incluso desde una posición casi parasitaria, como ocurre con el Tractatus logico-philosophicus de Wittgenstein. Éste, tras convertir la filosofía en análisis del lenguaje, subordinado a las ciencias (véanse las proposiciones 4.0031 y, sobre todo, 6.53), afirma lo siguiente: "Sentimos que aun cuando todas las posibles cuestiones científicas hayan recibido respuesta, nuestros problemas vitales todavía no se han rozado en lo más mínimo" (6.52).$^{87}$

La ancilaridad también puede ser necesaria por motivos sociales e individuales (e. g., políticos, científicos, técnicos, etc.), a veces de vida o muerte. La actitud socrática frente al Tribunal no fue filosófica porque se ocupara en el "taller" de las ideas durante su defensa, sino porque consideró que era mejor morir, y dar ejemplo a los atenienses, que vivir sin filosofía. La muerte de Sócrates, como ilustra la imagen de la Caverna, es un momento esencial de su filosofía, que define su racionalidad. Tampoco nos ha de extrañar que un filósofo como Platón, en la República (473d), proponga unir, en una sola persona, los poderes político y filosófico, aunque lo considere imposible, pero no a causa del filósofo, sino de quien no recurre a él (488a y ss.). Igualmente, las acepciones parasitarias de tipo fúnebre, e. g., la Carta sobre el Humanismo de Heidegger, al considerar que "el pensar futuro ya no es filosofía"; 88 Pour Marx (1965) de Althusser, al distinguir "filosofía" (ideológica) y "teoría" (científica), ${ }^{89}$ recordando de paso la tesis de tantos marxistas militantes, para quienes había que dejar de filosofar y pasar a la acción revolucionaria; ${ }^{90}$ o la conclusión de Stephen Hawking sobre la

${ }^{84}$ Ortega y Gasset: ¿Qué es filosofía?, p. 50.

${ }^{85}$ E. g., Ib., p. 65. Cf., p. 76 y p. 119.

${ }^{86} \mathrm{Ib}$., p. 69.

87 Wittgenstein, Ludwig. Tractatus logico-philosophicus, trad. de Jacobo Muñoz e Isidoro Reguera. Alianza, Madrid, 2010.

${ }^{88}$ Heidegger, Martin. Carta sobre el Humanismo, p. 90.

${ }^{89}$ Althusser, Louis. La revolución teórica de Marx, trad. de Marta Harnecker. Siglo XXI, Buenos Aires, 2004, p. 31.

${ }^{90} \mathrm{Ib}$., p. 20. 
"muerte de la filosofía”, al suponer que los filósofos ya no están al tanto de las últimas modas científicas, tienen al menos una virtud: al proclamar la muerte de la filosofía, hacen —a su pesar- filosofía (aunque sea mala), algo que Aristóteles ya recogió en su Protréptico: si filosofas, filosofas, y si no filosofas, filosofas para justificar que no filosofas, luego filosofas. ${ }^{91}$

El error de las acepciones rectas y oblicuas no es que se hayan equivocado de "contenido" (sin que tengamos criterios para saber por qué), sino que han seguido, con una actitud filosófica (podemos llamarla —en sentido estricto- "socrática"), el imperativo de ocuparse de las cuestiones más importantes, según las posibilidades de su presente. Sus obras no dejan por ello de ser filosóficas. La suposición de “causas finales” debía ocupar a los filósofos de su época, no porque persiguieran ocuparse de ideas y, de manera torpe, erraran en el intento, sino porque esas "causas" parecían importantes. La física, la poética, el comportamiento animal, no eran filosóficos para Aristóteles porque hubiese en ellos "ideas", sino porque el creador del Liceo consideró muy importante conocerlos.

\section{A modo de conclusión}

La obra de Bueno contiene muchos ejemplos de ideas importantes para nuestro presente: la Democracia, la Ciencia, la Religión, los Derechos Humanos, la Felicidad, la Televisión, la Cultura, la Izquierda, la Derecha, el Deporte. No parece haberlas elegido solamente porque sean ideas, sino también porque eran importantes. Pero igualmente importantes parecen los saberes de primer grado que, a menudo, explicaba Bueno en sus obras, o su modo de ser, del que sus discípulos, oyentes y lectores también aprendían a filosofar. Los imperativos de los filósofos (desde "Conócete a ti mismo" hasta "¡A las cosas mismas!”, pasando por el "Caute!” de Spinoza, el "Sapere Aude!" de Kant o su imperativo categórico) apelan a esa modalidad, a esa actitud crítica en el sentido expuesto. Bueno repitió alguna vez el adagio estoico: "El universo, mudanza; la vida, firmeza". ¿Y acaso estamos en condiciones de negar que su modo de firmeza es menos importante que su trato con ideas?

En otro lugar he argumentado que la importancia de los diálogos de Platón (el mayor homenaje que un filósofo ha hecho a otro filósofo), está en revivir la figura de Sócrates, no por sus doctrinas, sino por su ejemplo de vida. ${ }^{92}$ La mayéutica está en el centro de la filosofía crítica, y si ocupa

${ }^{91}$ Aristóteles. Protréptico, frs. 2-6.

${ }_{92}$ Martín Sáez, Daniel. “Eva Brann”, Ápeiron. Estudios de filosofía, no 4 (2016), pp. 161-71. 
ese lugar se debe más a su modalidad (determinada por el imperativo filosófico), que al ejercicio de martillear en el taller de las ideas. El imperativo "¡Dedícate a lo mejor!” puede tomarse como el imperativo implícito de todos los imperativos filosóficos: “Conócete a ti mismo!”, "Sapere aude!”, “¡A las cosas mismas!", están ya dando por supuesto que eso es lo mejor que uno puede hacer. En el ámbito de la filosofía helénica, no deja de resultar interesante que los denominados "sabios" —algunos de ellos (como Tales) involucrados en su momento primitivo-, se caracterizaran por imperativos de ese tipo: "Nada con exceso", "Sé previsor", etc. Las obras de Platón y Aristóteles están plagadas de ejemplos donde se dedica atención a una cosa u otra, o se decide qué modo de vida es mejor o peor, apelando a su importancia.

A esto sólo podría objetarse con una pregunta sofística: pero ¿por qué ser un filósofo? La pregunta es filosófica y supone una circularidad análoga a la señalada por Aristóteles en el Protréptico. Es como preguntar por qué hay que hacer preguntas, como si eso no fuera ya una pregunta, y por tanto una respuesta sobre la función del preguntar. La cuestión se resuelve de manera negativa. Es imposible dar una razón no-filosófica para no ser filósofo: el famoso "primum uiuere, deinde philosophari", es un oxímoron, como "círculo cuadrado". La respuesta de Sócrates a la pregunta sería dialéctica: no soy un sabio, ése no es mi modo de ser, y por tanto he de filosofar. "Sólo sé que no se nada" define la actitud filosófica por excelencia, el inicio del filosofar. La filosofía comienza con el reconocimiento de que la subjetualidad (según la acepción crítica) está atravesada de mitos, dogmas, saberes imperfectos en pugna, etc., y sólo podemos aspirar — como muestra el $\varphi$ íros de la palabra $\varphi \imath \lambda о \sigma o \varphi i ́ \alpha$ - a hacer las cosas lo mejor posible, sin temor a ser aficionados o equivocarnos, sin ser dogmáticos ni subordinar la filosofía o nuestra subjetualidad (en la medida de nuestras posibilidades) a otros saberes o subjetualidades, como si la filosofía fuera una actividad ancilar o parasitaria, o como si nosotros mismos fuésemos siervos o parásitos. La única cuestión relevante es si uno es filósofo o no lo es: si uno se ha dedicado o no a lo más importante, si ha sido capaz de ser lo mejor posible. Más allá de las Ideas o el principio de Symploké, los diálogos socráticos recogen ese particular modo de ser (subjetual) crítico, implantado, dialéctico, que la pregunta sofística ejerce, anulando la propia pregunta.

Quizá los filósofos se parezcan más por su modo de ser que por sus doctrinas. (Esto explicaría por qué muchos consideran decisivo el nazismo de Heidegger para condenarlo - esencialmente- como filósofo.) Lo esencial de la filosofía no es la geometría de las ideas. El momento crítico 
de la definición imperativa implica que al filósofo le interesa, en principio, todo, al menos por cuanto ignora siempre qué es lo mejor, sin perjuicio de que, para filosofar, uno deba entregarse desde el principio a esa geometría. En otras palabras, la geometría no es un fin, sino un medio del filosofar: uno, sin duda, del que sólo la filosofía sabe ocuparse con solvencia, pero insuficiente para agotar sus fuerzas. La filosofía, en su acepción crítica, no puede ser una especialidad. El fundador de la Academia consideraba a la

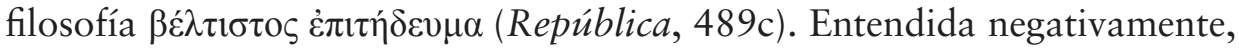
es una definición insuperable. La filosofía consiste en hacer lo mejor y es, por tanto, "la mejor ocupación".

\section{Bibliografía}

Althusser, Louis. La revolución teórica de Marx, trad. de Marta Harnecker. Siglo XXI, Buenos Aires, 2004.

Aristóteles: Protréptico, ed. de Carlos Megino Rodríguez. Abada, Madrid, 2006.

Bueno, Gustavo. El papel de la filosofía en el conjunto del saber. Ciencia Nueva, Madrid, 1970.

Bueno, Gustavo. Ensayos materialistas. Taurus, Madrid, 1972.

Bueno, Gustavo. "El concepto de 'implantación de la conciencia filosófica'. Implantación gnóstica e implantación política”, Revista de Occidente, Madrid, 1972, pp. 37-71.

Bueno, Gustavo. "Teorema entrevista a Gustavo Bueno". Teorema, vol. III, $\mathrm{n}^{\circ} 1$ (1973), pp. 123-40.

Bueno, Gustavo. Etnología y Utopía. Respuesta a la pregunta: ¿qué es la Etnología?. Serie Antropología, Júcar Universidad, 1987.

Bueno, Gustavo. ¿Qué es la ciencia? La respuesta de la teoría del cierre categorial. Ciencia y Filosofía. Pentalfa, Oviedo, 1995.

Bueno, Gustavo. ¿Qué es la filosofía? El lugar de la filosofía en la educación. El papel de la filosofía en el conjunto del saber constituido por el saber político, el saber científico y el saber religioso de nuestra época. Pentalfa, Oviedo, 1995.

Bueno, Gustavo. "Un profesor de filosofía, autor de libros de texto, en la década del 'nacional catolicismo' español”. El Catoblepas, no 99 (2010), p. 2. (http://nodulo.org/ec/2010/n099p02.htm, última consulta: 20/01/2017.)

Bueno, Gustavo. "Respuesta, en 1978, a la pregunta ¿Qué es el cierre categorial?". El Catoblepas, n ${ }^{\circ} 108,(2011)$, p. 2. (http://nodulo.org/ec/2011/ n108p02.htm, última consulta: 20/01/2017.) 
Bueno, Gustavo. "En torno a la distinción entre 'Conceptos' e 'Ideas'”. El Catoblepas, $\mathrm{n}^{\mathrm{o}} 127$ (2012), p. 2. (http://nodulo.org/ec/2012/n127p02. htm, última consulta: 20/01/2017.)

Bueno, Gustavo. Ensayo de una definición filosófica de la Idea de Deporte. Pentalfa, Oviedo, 2014.

Bueno, Gustavo. "Historia de las Ideas filosóficas". El Catoblepas, n 162 (2015), p. 2.

Bueno, Gustavo. "Prólogo a El papel de la filosofía en el conjunto del saber", El Catoblepas, n 173 (2016), p. 2.

Bueno, Gustavo. El Ego trascendental. Pentalfa, Oviedo, 2016.

Dilthey, Wilhelm. Esencia de la filosofía, trad. de Elsa Tabernig. Losada, 2003.

Copérnico, Nicolás. Sobre las revoluciones (de los orbes celestes), ed. de Carlos Minguez Pérez, Tecnos, Madrid, 2009.

Deleuze, Gilles \& Guattari, Félix. ¿Qué es la filosofía?, trad. de Thomas Kauf, Anagrama, Barcelona, 1997.

Diogenes Laertius. Lives of Eminent Philosophers, ed. de R. D. Hicks [1925], Harvard University Press, Cambridge, 1972.

Fernández Liria, Pedro. ¿Qué es filosofía?: Prólogo a veintiséis siglos de historia. Akal, Madrid, 2010.

Fichte, Johann Gottlieb. Versuch einer neuen Darstellung der Wissenschaftslehre. Felix Meiner Verlag, Hamburg, 1984. https://doi. org/10.28937/978-3-7873-2569-6

Gaos, José. Filosofía de la filosofía. FCE, Madrid, 2009.

Heidegger, Martin. ¿Qué es la filosofía?, trad. de Jesús Adrián Escudero. Herder, Barcelona, 2004.

Heidegger, Martin. Carta sobre el Humanismo, trad. de Helena Cortés y Arturo Leyte, Alianza, Madrid, 2006.

Marcus Aurelius. Ad se ipsum, ed. de Jan Hendrik Leopold. B. G. Teubneri, Leipzig, 1908.

Martín Sáez, Daniel. “Eva Brann”, Ápeiron. Estudios de filosofía, n 4 (2016), pp. 161-71.

Nudler, Oscar (ed.). Filosofía de la filosofía. Trotta, Madrid, 2010.

Ortega y Gasset, José. ¿Qué es filosofía? Ediciones de la Revista de Occidente, Madrid, 1976.

Sacristán Luzón, Manuel. "Sobre el lugar de la filosofía en los estudios superiores”. Editorial Nova Terra, Barcelona, 1968.

Unamuno, Miguel. Obras completas, X, ed. de Ricardo Senabre. Biblioteca Castro, Madrid, 2009. 
von Hildebrand, Dietrich. ¿Qué es la filosofía? [1973], trad. de Araceli Herrera, Encuentro, Madrid, 2000.

Wittgenstein, Ludwig. Tractatus logico-philosophicus, trad. de Jacobo Muñoz e Isidoro Reguera, Alianza, Madrid, 2010. 